\title{
Proceso de sistematización del trabajo de acercamiento comunitario en la
}

\author{
comunidad del Huaso de Aserrí \\ Shirley Broitman
}

\section{Universidad de Iberoamérica}

\section{Resumen}

Se realizó una intervención en una comunidad de escasos recursos, la cual se ha formado recientemente producto de un programa de vivienda popular; con el objetivo de ayudar a los miembros de esta nueva comunidad con la integración, organización, establecimiento de prioridades y resolución de problemas. Mediante talleres que abordaron los temas sugeridos por los miembros y considerados como los más importantes para estos se establecieron objetivos que permitieron categorizar las problemáticas por áreas, explorar los recursos con los que cuenta la comunidad, construir lineamientos generales de planificación y reflexionar sobre la importancia de la perseverancia. Se presentaron dificultades tales como, diferencias entre los participantes, el hecho de darse rápidamente por vencido al no lograr los objetivos, la falta de participación y empoderamiento junto con el desconocimiento del sistema burocrático. Se logró motivar a los miembros de la comunidad para participar y no depender o criticar a aquellos miembros que sí asumen tareas y responsabilidades, además del aprendizaje para lograr priorizar y organizarse. Para los estudiantes también significó un desafío y una experiencia valiosa en el proceso educativo. En la evaluación final la comunidad mostró satisfacción con las sesiones y lo aprendido.

Palabras clave: psicología, comunidad, taller

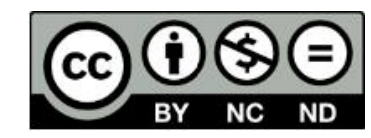




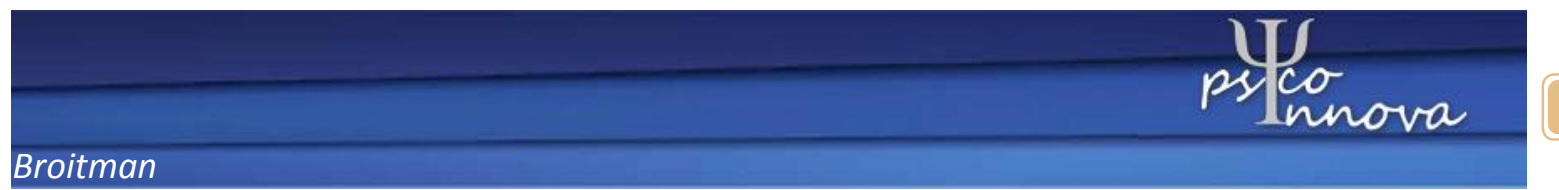

\section{Introducción}

En el primer cuatrimestre del 2016 el grupo de estudiantes del curso de psicología comunitaria de la UNIBE realizó una intervención en la comunidad del Huaso de Aserrí. La profesora a cargo del curso era la MPsc. Jeannette Castillo quien coordinó con las autoridades del FUPROVI el acceso a la comunidad. FUPROVI es una organización costarricense privada de desarrollo fundada en 1987, se encarga de apoyar a las familias de menores ingresos a solucionar sus necesidades habitacionales y de desarrollo comunal por medio de una participación activa de las misma familias estimulando la involucración de estas en la gestión, administración y construcción de las obras de infraestructura y vivienda de su proyecto habitacional (FUPROVI, 2013).

En el Huaso se ejecutó un programa de vivienda en donde confluyeron familias procedentes de la zona de Aserrí y del Triángulo de Solidaridad, lo que, al ser grupos provenientes de dos sectores diferentes, creó roces y conflictos interpersonales. A esta problemática se le intentó dar solución por medio de la psicoeducación, impartida en esa oportunidad, por el grupo de estudiantes de Unibe del último cuatrimestre del 2015, quienes bajo la supervisión de la profesora Castillo impartieron un taller sobre habilidades sociales y asertividad en la población de Huaso para mejorar la comunicación entre vecinos y lograr de este modo que la organización de los miembros de la comunidad fuera más fluida y adecuada.

Luego, durante el período de enero a mayo del 2016 se realizaron tres reuniones con los integrantes de la comunidad en donde después de realizar un diagnóstico se determinó que 
necesitaban ayuda en la organización, establecimiento de prioridades y orientación para resolver los problemas que se les presentaban (empoderamiento, funcionamiento de entes gubernamentales y aspectos jurídicos además de concientización sobre la participación de todos los miembros).

Los primeros aspectos a tomarse en cuenta antes de introducir a los estudiantes en el trabajo de campo se refieren a los conceptos teóricos necesarios para comprender diversas aristas de la piscología comunitaria. Primero, se trabajó con la definición de comunidad aportada por Montero, (2004) quien explica que, esta consiste en un grupo de personas que se encuentran en un constante cambio y desarrollo, en donde hay una relación directa entre los individuos, una identidad social y una consciencia de comunidad que los lleva a preocuparse por el resto de los que forman este grupo. Estas relaciones van forjando la unión y la interacción social entre los miembros y fortalecen este ente conocido como comunidad. Puesto que la comunidad del Huaso se había formado recientemente, algunos de estos factores se encontraban en proceso de maduración por ejemplo la identidad del grupo junto con la consciencia de orientar el trabajo hacia el bien común en vez del propio.

Por otro lado, era importante que los educandos percibieran el alcance de la psicología comunitaria y por ende su definición. Por tanto, se fundamentó la práctica por medio de enunciados de distintos autores, como es el caso de Montenegro, Montenegro, e Íñiguez, (2006) quienes citan:

"Bajo este nombre se abre un paraguas de diferentes prácticas y de relaciones posibles entre la “psicología” y “comunidad”. Quintal de Freitas (1994) sistematiza cuatro de las relaciones entre estas dos palabras.

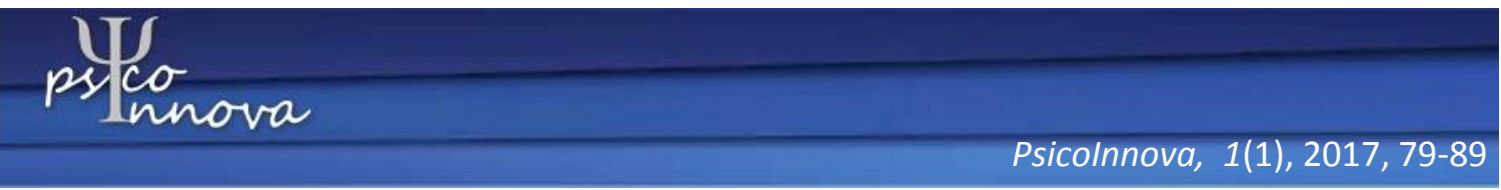




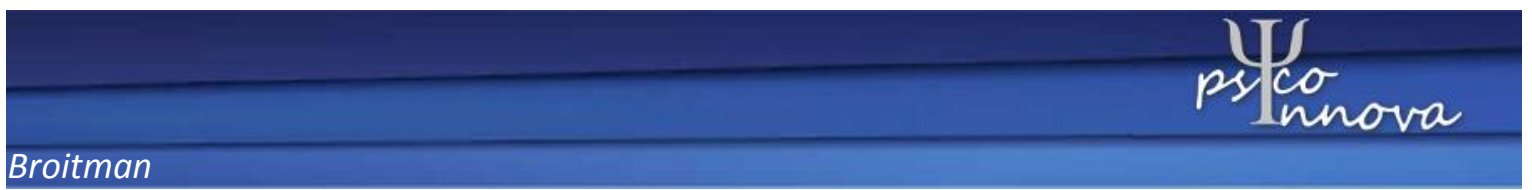

1. La psicología en la comunidad, la cual implica que se utilizan las herramientas psicológicas tradicionales en lugares donde no están fortalecidos los servicios de salud [...]

2. La psicología de la comunidad, en la cual los problemas son entendidos como derivados de los factores económicos, políticos y sociales de las sociedades y los profesionales se ponen al servicio de las demandas y necesidades de los grupos comunitarios como activismo político.

3. La psicología comunitaria en la cual se da una relación dialéctica entre personas de la comunidad y profesionales [...]" (p.64-65).

Y Montero (1984), quien define la psicología social como:

"[la] rama de la psicología cuyo objeto es el estudio de los factores psicosociales que permiten desarrollar, fomentar y mantener el control y poder que los individuos pueden ejercer sobre su ambiente individual y social, para solucionar problemas que los aquejan y lograr cambios en estos ambientes y en la estructura social" (p.390).

Ampliando por tanto, la tercera definición e indicando que los profesionales al practicar la psicología comunitaria son catalizadores que favorecen la transformación social al trabajar con las personas de la comunidad (Montero et al, 2006).

\section{Método}

Se trabajó con una población de escasos recursos de la comunidad del Huaso, la cual formó parte del programa de vivienda coordinado por FUPROVI. Consistía de 20 familias, la mayoría mujeres entre edades que van desde los 19 a los 70 años, además niños en edades entre 4 y 12 años. FUPROVI recomendó ayudar a la comunidad a hacer comisiones, pero se 
definió realizar en primera instancia un diagnóstico para definir las necesidades de la comunidad en ese momento.

Se realizaron 3 sesiones estilo taller de 1.5 horas en la Iglesia de la comunidad, en las cuales se implementaron diversas actividades con objetivos específicos para lograr la psicoeducación comunitaria.

El Taller fue denominado "Alcanzando metas comunes" donde los responsables de su ejecución fueron siete estudiantes del curso de psicología comunitaria de Unibe, en el primer cuatrimestre del 2016. La programación y las fechas de las sesiones se indican en la tabla 1, en un horario establecido los lunes de 1:30 - 3:30 p.m. Se lograron un total de 6 horas efectivas de trabajo. La comunidad constaba de treinta personas representantes de las familias que recibieron casas del INVU por medio de la organización FUPROVI.

Conforme se avanzaba en los talleres se establecieron los siguientes objetivos: general: Estructurar junto con la comunidad el proceso de resolución de problemas y los factores asociados a este (priorizar, planificar, resolver, reestructurar) y específicos: 1. Categorizar las problemáticas por áreas (hábitat, salud, educación, seguridad, recreación y deportes, otros) y priorizarlas. 2. Explorar los recursos con los que cuenta la comunidad para solucionar el problema de la basura y cómo han sido hasta el momento aprovechados. 3. Construir en conjunto con la comunidad lineamientos generales de los procesos de planificación. 4. Reflexionar acerca de la importancia de la perseverancia; cada uno de estos correspondientes a los diferentes talleres. 


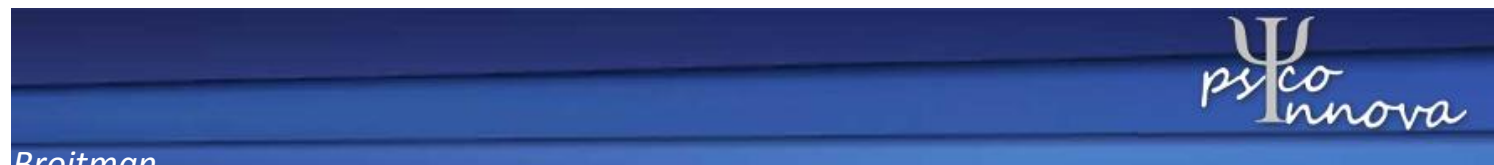

Broitman

Con base en el Trabajo de Acercamiento Comunitario de Broitman, Algom y Nielsen, (2016) se presenta a continuación la sistematización del proceso grupal desarrollado. Por tanto, en primera instancia se presenta una síntesis del contenido de cada una de las sesiones ejecutadas, tomando en cuenta aspectos tales como, objetivo, temática, actividades y técnicas a realizar. Seguidamente se muestran los resultados de cada sesión, y el análisis de las transformaciones dadas en el transcurso del proceso grupal.

Primera sesión

Participaron 22 adultos y 4 niños. Se inicia con la creación de reglas para el taller, con el objetivo de mantener el orden, bajo mutuo acuerdo durante las sesiones de trabajo. Estableciendo las siguientes reglas grupales: Respetar las opiniones y la palabra, participar en las actividades, ser tolerantes con los otros (ser paciente con lo que los otros decían), mantener los celulares en silencio o pagados, evitar las salidas del recinto para no interrumpir.

La segunda actividad llamada "la telaraña" buscaba la presentación del grupo y actuar a modo de rompe-hielo, además los participantes indicaban que era lo que más les gustaba de su comunidad. A la vez, se plantea una analogía sobre la telaraña y las relaciones entre la comunidad, pues cada vez que alguien hala uno de los hilos afecta al otro de alguna manera. Los participantes mencionaron lo que les gustaba: escuelas y parques cerca, vecinas buenas, servicio de bus, enamorada de la casa, panadería y carnicería cerca, cerca de familiares, buen servicio de Ebais, zona de recreación y zonas para socializar. La tercera actividad se denominó “explicación de conceptos” para diferenciar entre necesidad y problemática. La cuarta actividad llamada “desarrollar la capacidad de deducción de los problemas de la comunidad" buscaba categorizar los problemas que plantearan los miembros del grupo, 
posteriormente los participantes votaron para determinar cuáles problemas eran más urgentes, con la idea de colocarlos en "el semáforo", es decir, lo más urgente era rojo, lo intermedio amarillo y lo verde menos urgente. Por tanto se obtuvo: 1. Seguridad: a) la acumulación de gran cantidad basura en las aceras incluso días antes de que pase el camión recolector; b) "barras" de muchachos en las entradas de las casas. 2. Salud: a) la basura; b) caños traseros en donde se acumula el agua con el reisgo de reproducción de zancudos. 3. Hábitat: a) la basura; b) ruido de las "barras"; c) mantenimiento de los jardines. Y las prioridades determinadas por medio de "el semáforo" fueron rojo: caños y basura; amarillo: jardines y seguridad (drogas y barras) y verde: ruido (vecinos y grupos de muchachos). Se debió decidir entre los dos problemas en categoría roja, determinando la basura como el problema que más les afectaba.

Segunda sesión

Asistieron 20 adultos y 5 niños. Se recordaron las reglas de trabajo grupal como primera actividad. En la segunda actividad llamada "la basura en la comunidad" se determinó cuáles eran las causas del problema de la basura para luego poder buscar soluciones, se obtuvo de estos la siguiente información: a) causas del problema: no hay un lugar determinado para la basura por lo que los habitantes la dejan en un lote baldío, se deja en horarios en los que el camión recolector no pasa, el camión no ingresa a las alamedas; b) acciones que ya se han realizado para intentar solucionar el problema: se ha hablado con algunos vecinos, miembros del grupo fueron a la municipalidad de Aserrí donde, de acuerdo con ellos, les solicitaron hacer una carta anónima (para no tener problemas con los vecinos) explicando el problema; c) obstáculos para resolver el problema: los miembros del grupo no

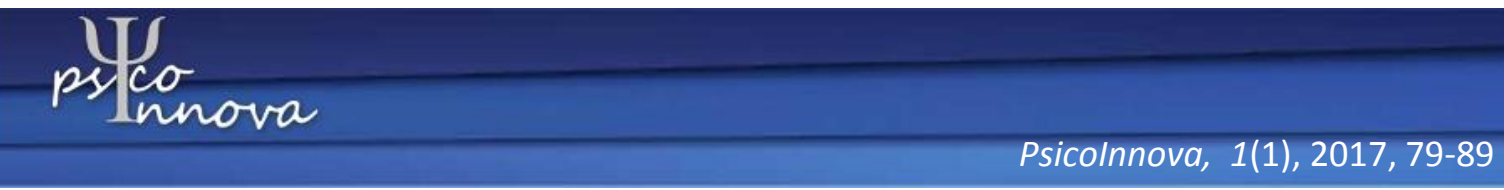




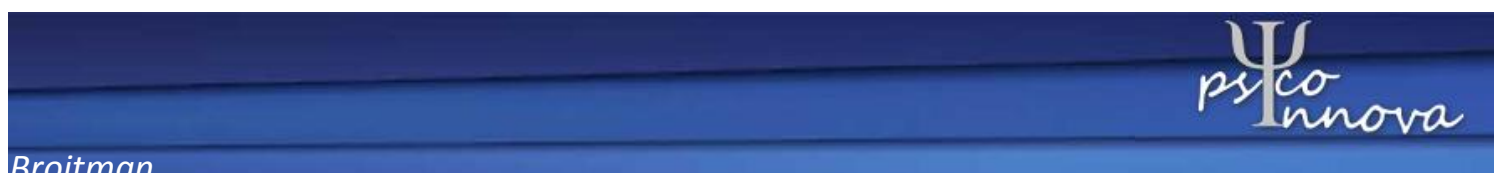

quieren pelearse o caerle mal a los vecinos que sacan la basura a destiempo, habían unos estañones para poner la basura pero fueron robados, el lote donde se pone la basura no le pertenece a nadie, no hay consciencia de la importancia de la limpieza, falta de apoyo entre todos; y d) si la problemática se mantiene: se afecta la salud por los mosquitos y perros, hay que resignarse. En la tercera actividad, llamada "explorando los recursos", se definieron los recursos que dispone la comunidad para resolver el problema de la basura. Para esto se monta una tabla en el piso de la Iglesia con cuadros de cartulina en donde se incluyen objetivo, encargado y plazo (tabla 2). Asimismo, se exploraron los recursos humanos, financieros, materiales y organizacionales disponibles (tabla 3) con la idea de que sus miembros descubran las diversas posibilidades con las que pueden contar para resolver el problema y a la vez, estipular en conjunto cual es la mejor manera de acceder a ellas y descubrir que algunas son de fácil acceso.

\section{Tercera sesión}

Participaron 15 adultos y 2 niños. Como primera actividad se recordaron nuevamente las reglas, además se hizo énfasis en el aspecto de la participación y la importancia de esta durante la sesión, como forma de promover la participación de los integrantes durante la sesión. En la segunda actividad, "armando la realidad” se exploró el avance de las tareas asignadas encontrándose que sí se realizaron cinco de ocho tareas, lo cual representa un gran avance y demostrando que la asignación de tareas realizadas fue exitosa. En la tercera actividad llamada "reestructuración de tareas" se determinó y ordenó la estructura de las tareas pendientes y el seguimiento de las ya avanzadas, junto con la indagación de la posible solución. Asimismo, se presentó mucha dificultad por parte de los participantes para 
responsabilizarse por alguna de las tareas, por lo que se requirió designarlas bajo presión. Se logró así que casi todos asumieran alguna responsabilidad, lo cual fue de suma importancia para que se comprendiera la importancia que tienen todos como equipo con respecto a lo que le sucede a su comunidad. La cuarta actividad "el bambú japonés” consistió en una reflexión que estimulaba la importancia del trabajo constante y consistente para lograr las metas.

Evaluación

Al final del taller se realizó una evaluación la cual generó que de los 15 participantes, 8 valoraron el taller como muy bueno, en función de los siguientes rubros: los temas en sí, comprensión de los temas, actividades, calidad y capacidad de los expositores y la relación expositor-comunidad, además de los materiales utilizados. Ante la pregunta de que aprendí con el taller las respuestas indicaban: ser perseverante, tener comunicación, escuchar, sabiduría, apoyo para la comunidad, la convivencia, unidad, aprendizaje sobre la basura, organización en la comunidad, cosas importantes para el diario vivir.

\section{Conclusiones}

Producto del taller se logró categorizar las problemáticas de la comunidad estableciendo la acumulación de basura en las aceras como la situación prioritaria a resolver. Asimismo, determinó que los recursos con los que cuenta son los dirigentes comunitarios, la psicóloga de la escuela, los entes gubernamentales y organizaciones, recurso humano financiero y material, además del organizacional. Se construyeron en conjunto con la comunidad lineamientos de procesos de planificación en los cuales se asignaron las tareas por llevar a

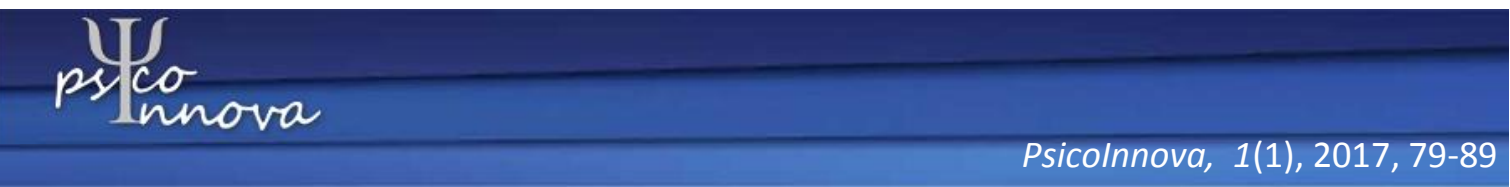




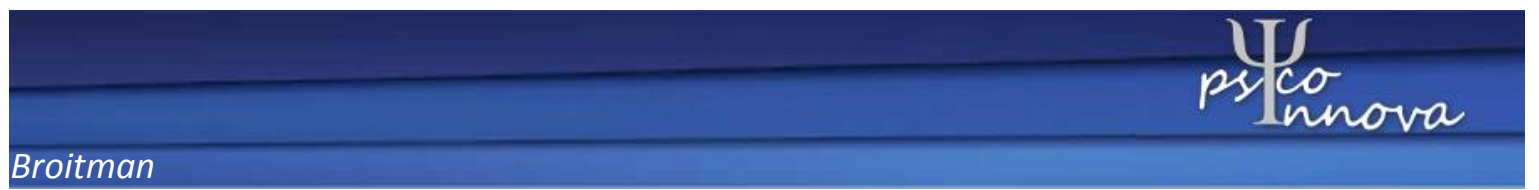

cabo, junto con sus responsables y los plazos para realizarlas. Se realizó una verificación de las tareas llevadas a cabo y aquellas que no se realizaron, basados también en determinar los obstáculos que impidieron su ejecución. Se reestructuraron aquellas actividades pendientes y se asignaron nuevos responsables, intentando la participación de todos. Por medio de la lectura de un cuento se reflexionó brevemente acerca de la perseverancia, la paciencia y el trabajo en equipo como herramientas valiosas para resolver los problemas a los que se enfrenta la comunidad. Es visible la falta de participación de los miembros de la comunidad para resolver sus problemas, en parte, por considerarse incapaces de afrontar los obstáculos, por falta de empoderamiento y por desconocimiento del sistema burocrático, el cual deben enfrentar constantemente. Asimismo, se observaron fricciones entre los miembros de la comunidad por lo que se enfatizó la importancia del trabajo en equipo.

Se logró por medio del trabajo en conjunto con la comunidad y los estudiantes estructurar un proceso que permitió a la comunidad priorizar sus problemas y necesidades, planificar los pasos para resolverlos, solucionar los obstáculos que se presentaron y reestructurar, en función de estos, las tareas a realizar para acercarse a su objetivo. De esta forma los miembros han adquirido herramientas básicas para organizarse mejor cuando no se encuentren bajo la guía de un coordinador. Por otro lado, es preocupante la "resignación” de los participantes cuando no logran sus metas.

Por tanto, se recomienda seguir implementando talleres psicoeducativos en la comunidad para mejorar el empoderamiento, trabajo en equipo, comunicación asertiva y organización. Asimismo es importante fortalecer las relaciones sociales y la motivación. Educar en temas de derechos civiles, sociales y administrativos de las entidades gubernamentales relacionadas 
con la comunidad permitiría mayor confianza y seguridad a la hora de realizar los trámites que dichos entes requieren. Propiciar la valoración de los voluntarios y el trabajo que realizan permitiría que las críticas fueran constructivas y favorecería la integración y las buenas relaciones. Junto con concientizar sobre la perseverancia ante la adversidad.

Asimismo, el aprendizaje fue también para los estudiantes ya que fueron acercados a una realidad lejana a la suya y debieron lidiar con los inconvenientes de la práctica de campo que no se darían si el contexto fuese solo teórico.

\section{Referencias}

Broitman, S., Algom, N. y Nielsen, I. (2016). Trabajo de Acercamiento Comunitario. Taller Alcanzando Metas Comunes. Tibás, San José.

FUPROVI. (2013). FUPROVI. Vivienda Social y de Desarrollo Comunal. Recuperado el 30 de junio de 2016, de http://www.fuprovi.org/

Montenegro, M., Montenegro, K. y Íñiguez, L. (2006). Acción comunitaria desde la psicología social. En X. Úcar, \& A. Llena, Miradas y diálogos en torno a la acción comunitaria (págs. 57-88). España: Graó.

Montero, M. (1984). La psicología comunitaria: orígenes, principios y fundamentos teóricos. Revista Latinoamericana de Psicología, 16(3).

Montero, M. (2004). Introducción a la psicología comunitaria. Desarrollo, conceptos y procesos. Editorial Paidós. Buenos Aires. Argentina. 